\title{
Lesser degrees of explanation: further implications of F. A. Hayek's methodology of sciences of complex phenomena
}

\author{
SCOTT SCHEALL \\ Arizona State University
}

\begin{abstract}
F. A. Hayek argued that the sciences of complex phenomena, including (perhaps especially) economics, are limited to incomplete "explanations of the principle" and "pattern predictions". According to Hayek, these disciplines suffer from-what I call-a data problem, i.e., the hopelessness of populating theoretical models with data adequate to full explanations and precise predictions. In Hayek's terms, explanations in these fields are always a matter of "degree". However, Hayek's methodology implies a distinct theory problem: theoretical models of complex phenomena may be underspecified so that, even when all data is available, a full explanation could not be inferred from the model. Where the sciences of complex phenomena are subject to both the data and theory problems, explanations and predictions will be of even lesser "predictive degree". The paper also considers how to interpret Hayek's claim that pattern predictions are falsifiable.
\end{abstract}

Keywords: Hayek, explanation, prediction, economics, methodology, complexity

JEL Classification: A12, B31, B41, B53

From the early-1950s on, F. A. Hayek was concerned, among his several other interests, with the development of a methodology of sciences that study systems of complex phenomena. ${ }^{1}$ According to Hayek, complex

\footnotetext{
1 The various disciplines that Hayek counted under the heading of sciences of complex phenomena include theoretical psychology (1952), "cybernetics, the theory of automata or machines, general systems theory, and perhaps also communications

AUTHOR's NoTE: This project was completed while I was a research fellow with Duke University's Center for the History of Political Economy. Many thanks to Bruce Caldwell, Gene Callahan, Catherine Herfeld, Joseph Ranweiler, Solomon Stein, two anonymous reviewers, and Luis Mireles-Flores (EJPE co-editor) for their several constructive comments and criticisms. All remaining errors of omission and commission are, unfortunately, my own.
} 
phenomena consist of a large number of elements interconnected (both to each other and to the external environment) in such a way as to give rise to an emergent order that possesses "certain general or abstract features which will recur independently of the particular values of the individual data, so long as the general structure [...] is preserved" (Hayek 1967 [1964], 26). The scientist of complex phenomena investigates these emergent orders and their properties, which cannot be fully reduced to the properties of the particular elements involved (Hayek 1967 [1964], 39). However, the knowledge that can be acquired about such orders is limited-in virtue of their complexity (and the comparatively narrow boundaries of human cognitive faculties)-as compared to the knowledge that scientists of simpler phenomena can acquire about the objects of their analyses. In particular, Hayek (1967 [1964], 27) argued that the number of elements of such complex systems is so large as to constrain the capacity of the scientist of complex phenomena to populate theoretical models with data sufficient to generate any but circumscribed explanations ("explanations of the principle") and predictions ("pattern predictions"). ${ }^{2}$

The present paper aims to draw out and clarify a number of further implications of Hayek's methodology of sciences of complex phenomena that have heretofore been unspecified in the primary and secondary literature on Hayek. In particular, the paper seeks to elucidate the implications of Hayek's methodology with respect to the specific dimensions along which the scientist's knowledge of some complex phenomena may be limited. Hayek's fallibilism-i.e., the epistemological position according to which knowledge is never complete and, in any case, always revisable in the light of new evidence-was an essential (if not always explicit) aspect of his arguments against the defenders of both socialism (1948 [1935]; 1948 [1940]) and countercyclical monetary policy (1978 [1975]). Yet, despite the fact that his conceptions of both complex phenomena and the methodology appropriate to their investigation imply that ignorance might beset the scientist in respects beyond the aforementioned difficulties of data collection, he never explicated these latter implications of his methodology.

theory" (1967 [1955], 20); as well as economics, linguistics (1967, 72), geology, evolutionary biology, and the branches of astrophysics that investigate the formation of stars and galaxies $(1967,76)$.

${ }^{2}$ Hayek (1967 [1955], 9n) understood explanation and prediction to be two sides of the same coin. The two terms are used interchangeably here. 
Predictive capacities are limited wherever such ignorance is rife. More to the point, the specificity of a scientific prediction depends on the extent of the relevant scientist's (or scientific community's) knowledge concerning the phenomena under investigation. The paper offers an account of the considerations which, according to Hayek's methodology, determine the extent to which a theory's implications prohibit the occurrence of particular events in the relevant domain. This theory of "predictive degree" both expresses and-as the phenomena of scientific prediction are themselves complex in Hayek's senseexemplifies the intuition that the specificity of a scientific prediction depends on the relevant knowledge available.

\section{I}

According to Hayek's epistemology, knowledge comes in two varieties: there is "scientific" (or "theoretical") knowledge ("knowledge of general rules") and there is empirical knowledge ("knowledge of the particular circumstances of time and place") (Hayek 1948 [1945], 80). ${ }^{3}$ The possibility of a "full explanation" or a "precise prediction of particular events" ${ }^{4}$ requires that the scientist possess both kinds of knowledge to a sufficient extent: "[s]uch prediction will be possible if we can ascertain [...] all the circumstances which influence those events. We need for this both a theory which tells us on what circumstances the events in question will depend, and information on the particular circumstances which may influence the event in which we are interested" (2014 [1961]). ${ }^{5}$

\footnotetext{
${ }^{3}$ Hayek's epistemology includes knowledge of which we may not be "explicitly aware", but which we "merely manifest [...] in the discriminations which we perform" (Hayek 1952, 19). This is "tacit" knowledge (Polanyi 1966) or "knowledge how" as opposed to "knowledge that" (Ryle 1946). Tacit knowledge is not, for Hayek, a third class of knowledge. Rather, we can both have tacit knowledge of general rules of conduct and only tacitly recognize the particular circumstances in which these general rules are relevant.

${ }^{4}$ A "full" explanation need not be complete in the sense of encompassing every detail of the phenomena under investigation: an explanation "can never explain everything to be observed on a particular set of events" (Hayek 1952, 182). The concept of explanatory "fullness" should be thought of as sensitive to scientific context.

${ }^{5}$ In addition to those sciences of complex phenomena mentioned in footnote 1 above, Hayek (1967 [1964]) offers two specific examples of theories of complex phenomena, namely, Darwin's theory of evolution by natural selection-“[p]robably the best illustration of a theory of complex phenomena which is of great value, although it describes merely a general pattern whose detail we can never fill in" (1967 [1964], 31) - and Walrasian general equilibrium theory, with respect to which Hayek writes:

[...] economic theory is confined to describing kinds of patterns which will appear if certain general conditions are satisfied, but can rarely if ever derive from this knowledge any predictions of specific phenomena. This is seen most clearly if we
} 
Hayek defines pattern predictions as the implications of theories that would suffice to generate detailed predictions of particular events if only the parameters ${ }^{6}$ of the theory could be filled in with sufficient empirical data: "[e]very algebraic equation or set of equations defines in this sense a class of patterns, with the individual manifestation of this kind of pattern being particularized as we substitute definite values for the variables" (1967 [1964], 24). Hayek writes that

[s]uch a theory[,] destined to remain 'algebraic', because we are in fact unable to substitute particular values for the variables, ceases then to be a mere tool and becomes the final result of our theoretical efforts. Such a theory...enables us to predict or explain only certain general features of a situation which may be compatible with a great many particular circumstances [...] [I]n many fields this will be for the present, or perhaps forever, all the theoretical knowledge we can achieve (Hayek 1967 [1964], 28-29). ${ }^{7}$

Thus, Hayek's definition of a pattern prediction implies that, other things equal, the degree of a prediction-i.e., the specificity "of the events mentioned [...] in the prognosis" (Hayek 1967 [1955], 8)-is positively related to the extent of the available data. ${ }^{8}$ We can also say that, a maximally-specific prediction, i.e., a prediction that rules out the occurrence of every possible event in the relevant domain but one (if you like, a prediction of degree 1 or, in Hayek's verbiage, "a precise

consider those systems of simultaneous equations which since Léon Walras have been widely used to represent the general relations between the prices and the quantities of all commodities bought and sold. They are so framed that if we were able to fill in all the blanks...we could calculate the prices and quantities of all the commodities. But, as at least the founders of this theory, clearly understood, its purpose is not [quoting Pareto 1927, 223-224] 'to arrive at a numerical calculation of prices', because it would be 'absurd' to assume that we can ascertain all the data (Hayek 1967 [1964], 35).

${ }^{6}$ Hayek uses three different terms seemingly interchangeably to speak of the theoretical elements of explanations, namely, "variable", "element" (1967 [1955]), and "parameter" (1964 [1967]). For the most part, I have adopted the latter locution, though I may occasionally use one of the other two terms merely to avoid excessive repetition.

${ }^{7}$ Also see Hayek $(1952,183)$ : "[t]he distinction between the explanation of the principle on which a wide class of phenomena operate and the more detailed explanation of particular phenomena is reflected in the familiar distinction between the 'theoretical' and the more 'applied' parts of the different sciences".

${ }^{8}$ Relatedly, predictive degree increases (other things equal) as the possible range within which the value of some variable might lie is narrowed. This is particularly relevant given the well-known vagaries of empirical measurement: ceteris paribus, the degree of a prediction increases as observational error is minimized (see Hayek 1967 [1955], 9-10). 
prediction of particular events"), is an implication of a fully-specified theory, and a complete (relative to the specified theoretical parameters) and precisely-measured data set. Stated more plainly, according to Hayek's epistemology, a scientist will be able to precisely predict particular events only if she knows everything of a theoretical and empirical nature relevant to the phenomena in the given context.

Moreover, Hayek's methodology of sciences of complex phenomena implies that for every theory (T) adequate to imply precise predictions of particular events, there is some number greater than 0 (the degree of a statement devoid of empirical content) and less than 1 (the degree of a precise prediction of particular events), which is the degree of what we might call a mere pattern prediction, i.e., an implication of the conjunction of an empty data set and a theory that is completely specified relative to the requirements of a full explanation in the relevant scientific context. Naturally, other things equal, the predictive degree of such a conjunction approaches 1 as the data set is increasingly populated.

Summarizing, a bit more formally, a precise prediction of particular events specified to the extent desired in the given scientific context requires both knowledge of a (positive and "large") number $p$ of theoretical parameters and knowledge of the particular value that each of these parameters assumes at the time relevant to the prediction. If (and only if) the scientist's knowledge satisfies both conditions, then her prediction will be of degree 1 . However, if the first condition is satisfied, i.e., if the scientist possesses knowledge of $p$ theoretical parameters, but she does not know the value of any of the parameters at the time relevant to the prediction, then she will only be able to make a mere pattern prediction, the degree of which will be greater than 0 and less than 1, but which will approach 1 as she acquires more knowledge of the relevant values of each of the $p$ parameters.

\section{II}

There are places in Hayek's methodological writings where he points to the fact that the case in which the scientist possesses an adequate theoretical understanding of the order under investigation (and, so, merely requires sufficient data in order to generate a precise prediction of particular events) is not the norm in the sciences of complex phenomena. As a matter of fact, there are many cases in which, relative to what would be required to generate a precise prediction, the 
scientist's theoretical knowledge is deficient, i.e., where the relevant "algebraic equation or set of such equations [that] defines [...] a class of patterns" (Hayek 1967 [1964], 24) is itself underspecified-contains gaps or lacunae with regard to the parameters required of a full explanation of the order under investigation-so that a precise prediction of particular events could not be generated even if the scientist possessed all of the relevant data. ${ }^{9}$

That Hayek took this theory problem (as distinct from the data problem he so thoroughly explicated) to be common in sciences of complex phenomena is implicit in the argument of "The dilemma of specialization" (1967 [1956], 124), ${ }^{10}$ an essay in which explicit methodological considerations take a backseat to concerns of best pedagogical practices in the social sciences. Hayek's claim that preeminence in these disciplines requires learning well beyond a narrow field of specialization is worth quoting at length as it gets to the heart of the theory problem implied by his methodology. "For almost any application of our knowledge to concrete instances", Hayek writes,

[...] the knowledge of one discipline, and even of all the scientific knowledge we can bring to bear on the topic will be only a small part of the foundations of our opinions. Let me speak first of the need of using the results of scientific disciplines other than our own, though this is far from all that is required. That concrete reality is not divisible into distinct objects corresponding to the various scientific disciplines is a commonplace, yet a commonplace which severely limits our competence to pronounce as scientists on any particular event. There is scarcely a phenomenon or event in society with which we can deal adequately without knowing a great deal of

\footnotetext{
${ }^{9}$ See, e.g., "A new look at economic theory", the first of four lectures delivered at the University of Virginia in 1961 (and now published as Hayek 2014 [1961]): "[e]ven a true theory will not enable us to make predictions of specific events unless we are able to ascertain all those relevant facts [...] which govern the particular position" [italics added]. One is left to ponder the significance of predictions of theories that might be "true" as far as they go, but which do not go very far because their theoretical parameters are not fully specified.

${ }^{10}$ Hayek's concern for the theory problem is also implicit in his criticisms of Keynes's Treatise on money (1971 [1930]) in the early 1930s. The main point that Hayek persistently pushed in his multi-part review (1995 [1931a]; 1995 [1931b]; 1995 [1932]) of the Treatise was the absence of any theoretical account of capital in the book. In his rejoinder, Keynes (1973 [1931], 252-253) accepted that a treatment of capital would figure in a perfected theory of a money-using economy, but insisted that the theory presented in the Treatise was adequate for his purposes at the time. Hayek and Keynes obviously had different conceptions of the requisite "fullness" of a satisfactory explanation of a money-using economy (or misconceived each other's scientific purposes).
} 
several disciplines, not to speak of the knowledge of particular facts that will be required (Hayek 1967 [1956], 124; italics added).

In other words, a theory capable of generating a full explanation of some complex phenomena may well be a composite system of theories, spanning multiple disciplines each of which might investigate phenomena of greater or lesser complexity. ${ }^{11}$ What is more, given that "concrete reality is not divisible into distinct objects corresponding to the various scientific disciplines", there may be phenomena that contribute to the emergence of a particular order and that must be accounted for if an explanation is to be "full", which fall under the heading of no extant scientific discipline. The relevant theoretical knowledge may not have been discovered (indeed, it may not even be discoverable).

Hayek (1967 [1964], 40-42) argued in the "Postscript" that accompanied later versions of "The theory of complex phenomena" that the ordinary notion of a scientific law, i.e., of a relation between cause and effect, has a clear meaning (whatever it might be) only with respect to "those two-variable or perhaps three-variable problems to which the theory of simple phenomena can be reduced". However, with regard to more complex phenomena,

[i]f we assume that all the other parameters of such a system of equations describing a complex structure are constant, we can of course still call the dependence of one of the latter on the other a 'law' and describe a change in the one as 'the cause' and the change in the other as 'the effect'. But such a 'law' would be valid only for one particular set of values of all the other parameters and would change with every change in any one of them. This would evidently not be a very useful conception of a 'law', and the only generally valid statement about the regularities of the structure in question is the whole set of simultaneous equations from which, if the values of the parameters are continuously variable, an infinite number of particular laws, showing the dependence of one variable upon another, could be derived (Hayek 1967 [1964], 41-42).

\footnotetext{
${ }^{11}$ The case in which a full explanation requires theoretical input from a number of disciplines illustrates the theory problem in all its ignominy, but the problem can just as well manifest in sciences where self-contained explanations are possible. All that is necessary (and sufficient) for the theory problem to arise is that, relative to the requirements of a full explanation of the phenomena under investigation, there be gaps in the specification of the parameters of the theory (or composite system of theories) meant to generate such an explanation.
} 
In other words, the individual parameters are not, strictly speaking, causes in the sense in which we normally think of them in explanations of simpler phenomena. The "cause" of an order is the whole network of parameters and particular values from which it emerges, and to know the cause of some order is to possess knowledge of all of these theoretical and empirical considerations.

That a scientist's theoretical understanding of some complex phenomena may underwhelm the requirements of a precise prediction of particular events-regardless of the availability of the relevant dataimplies that there are, on Hayek's methodology of sciences of complex phenomena, degrees of prediction less than the degree of mere pattern predictions. Consider a system of theories ( $T$ ) fully specified in terms of $p$ theoretical parameters for which no data are available, i.e., a system of theories capable of generating only mere pattern predictions. Now, imagine removing one of the parameters. The result of this excision $\left(T^{\prime}\right)$, which consists of $p-1$ parameters, will, other things equal, imply a pattern that is missing at least one of the parts of the pattern implied by $T$. A system of theories that would be capable of generating mere pattern predictions were its parameters fully specified will describe only partial patterns. Moreover, if we remove another parameter from $T^{\prime}$, the result $\left(T^{\prime \prime}\right)$, consisting of $p$-2 parameters, will, ceteris paribus, imply a pattern that is missing at least one of the parts of the pattern implied by $T^{\prime}$, and so on.

Indeed, a point will come in the process of eliminating parameters where the remaining variables will not imply anything like a substantive explanation that might be of interest to a scientist. ${ }^{12}$ That is, for any complex phenomena the scientist might want to explain, there is a minimum positive number of theoretical parameters $(p-n$, where $p$ is positive and "large", and $n<p$ ) she must know in order for her theory to express even minimal empirical content. The predictive degree associated with the $T^{n}$ that includes $p$-n parameters, the minimum number of parameters required for empirical content, sets a lower bound to the degree of associated partial pattern predictions. If the

\footnotetext{
${ }^{12}$ See Hayek $(1952,180)$ : "In general, the possibility of forming a model which explains anything presupposes that we have at our disposal distinct elements whose action in different circumstances is known irrespective of the particular model in which we use them". Similarly, the possibility of even limited explanations requires that we have at our disposal enough of these elements. Hayek (1967 [1964], 26) refers to the "minimum number of distinct variables a formula or model must possess in order to reproduce the characteristic patterns of structures of different fields (or to exhibit the general laws which these structures obey)".
} 
scientist knows fewer parameters than this, her theory cannot generate even partial pattern predictions, i.e., the theory's predictive degree is 0 .

\section{III}

It follows from the considerations adduced thus far that, ceteris paribus, the predictive degree of any theory $\left(T^{m}\right.$, where $\left.0 \leq m \leq n\right)$ consisting of $p$ - $m$ parameters, will be greater than or equal to that of $T^{n}$ and approach that of $T$ as more relevant parameters are added to the system. Of course, for any such $T^{m}$, predictive degree will, other things equal, increase as the $p-m$ parameters are increasingly populated with data. It also follows that, if we possess certain data points only some of which have clear theoretical interpretations with respect to the phenomena under investigation-that is, if we have some observations the theoretical significance of which is undetermined in the relevant context-then, as we are subsequently able to interpret these values in terms of (i.e., assign them to) particular parameters, the degree of associated predictions will increase (ceteris paribus).

However, an element of indeterminacy enters the frame when we consider the problem of comparing the predictive degrees of two (or more) conjunctions of theoretical and empirical knowledge, both concerning the same complex phenomena, one of which consists of a larger collection of parameters for which there are less data available and another that consists of a smaller collection of parameters for which there are more data available. To see this, consider the extreme case of comparing the predictive degree of a mere pattern prediction, i.e., the conjunction of $p$ parameters (which would suffice for a precise prediction if all of the data were available) and an empty data set, with the predictive degree of a conjunction of $p$ - $n$ parameters (the minimum number of parameters required for empirical content) and a set of values each of which can be assigned to one of the $p$ - $n$ parameters. Which of these conjunctions of theoretical and empirical knowledge has the higher predictive degree, i.e., which prohibits the occurrence of more events in the relevant domain? The situation here is that the first conjunction implies no details about a complete pattern of events while the second implies all of the details about a partial pattern of events. Whether this means that the predictive degree of the first conjunction is greater or lesser than that of the second is undecidable on the basis of a priori considerations alone. 
In order to determine the predictive degrees of two rival conjunctions of theory and data, one consisting of both more parameters and less data than its competitor, we need other theories (more precisely, we need meta-theories or second-order theories) that tell us how, for each rival conjunction, theoretical and empirical knowledge interacts so as to influence the degree of associated predictions. Stated another way, these meta-theories would be multi-valued functions that take as inputs "measurements" of the extent of theoretical parameters and data, and return specific, quantitatively-precise, degrees of prediction. This is to say that, at the meta-level, the relevant data would be the rival conjunctions of (first-order) theories and their respective data. Our problem is precisely that, in the abstract, without these rival first-order theories and their respective data, nothing about the shapes of the relevant multi-valued functions can be established by way of philosophical analysis alone.

All of this is just a way of saying that the theory of predictive degree implied by Hayek's methodology of sciences of complex phenomena is itself subject to both the data problem and the theory problem, and, therefore, cannot approach a full explanation of the epistemological aspects of predictions in the sciences of complex phenomena. However, this result is a strength rather than a weakness of both Hayek's methodology and the present attempt to elucidate the latter further. It means, in essence, that the phenomena of predictive degree are themselves complex and, thus-in concert with Hayek's methodologythat we are effectively limited by the complexity of these phenomena to an explanation of the principle of explanations of the principle. It is of course consistent for an explanation according to which explanations are limited to itself be limited. Indeed, the real threat to Hayek's methodology would be a full explanation of the phenomena of predictive degree, for this would signify either that these phenomena are not complex in Hayek's sense-a possibility which the slightest bit of reflection reveals to be unlikely-or that Hayek's methodology is internally inconsistent, i.e., that full explanations of complex phenomena are possible.

\section{IV}

To this point, I have relied on an implicit simplifying assumption to the effect that knowledge of any particular theoretical parameter or data point is equally important to a theory's predictive degree. That is, I have 
assumed that if a full explanation of some complex phenomena requires knowledge of (a positive and "large") number $p$ of parameters and each of their values, then knowledge of any $p$ - 1 of these parameters and their values would result in predictions of the same degree. However, there is nothing in Hayek's writings on complex phenomena that licenses this assumption. It may well be that knowledge of a certain collection of $p-m$ (where $0<m \leq n$ ) parameters and their values results in predictions of greater (or lesser) degree than knowledge of some other group of $p-m$ parameters and their values. Indeed, it is even possible on Hayek's methodology that knowledge of some collection of $p$-l (where $l>m$ ) parameters and their values results in predictions of greater degree than knowledge of a group of $p-m$ parameters and their values. In other words, it can matter for predictive degree not only how many items of theoretical and empirical knowledge the scientist possesses, but which pieces of knowledge she possesses, some items being more important for an adequate explanation of the emergent order than others. The scientist might possess fewer pieces of more important knowledge and generate predictions of greater degree than if she possessed more items of less important knowledge.

To see this latter point, consider again the quote above from "The dilemma of specialization". Presumably, what Hayek has in mind when he says that "the knowledge of one discipline, and even of all the scientific knowledge we can bring to bear on the topic will be only a small part of the foundations of our opinions" (1967 [1956], 124) is that a full explanation of, say, some economic phenomena, requires more knowledge than can be furnished by economic science alone-we would also need, e.g., historical, sociological, anthropological, and psychological knowledge, not to mention some knowledge provided by the physical and biological sciences. One would think-although, for the reasons stated in section III above, no proof can be offered-that the knowledge provided by economic science alone which, without any knowledge of these other disciplines, would represent a comparatively small proportion of the parameters required for a full explanation, would nonetheless yield a fuller explanation of the relevant economic phenomena than an explanation built entirely on knowledge of these other disciplines without any input from economics.

It should be obvious that dropping the simplifying assumption that knowledge of any particular theoretical parameter or data point is equally important leaves our theory yet further removed from a full 
explanation of the phenomena of predictive degree. Nonetheless, the considerations adduced thus far do license some very general statements about the effect of ignorance of relatively important theoretical parameters or their respective data on the degree of associated predictions. The partial pattern prediction implied by a system of theories missing relatively important parameters will, other things equal, describe less of the complex phenomena than an otherwise identical system missing the same number of less important parameters. To see this, return briefly to the example in the previous paragraph: it seems that, other things equal, the system missing $m$ economic parameters must predict less of the relevant pattern of economic phenomena under investigation than another system missing $m$ non-economic parameters. The former will imply lesser degree partial pattern predictions than the latter. Similarly, if the data associated with particularly important parameters are missing, then the resulting conjunction of parameters and data will imply the same pattern as an otherwise identical conjunction that happens to be missing the same number of data points associated with less important parameters; however, other things equal, the former will imply less of the more important details and more of the less important details of this pattern of events, while the latter will imply more of the more important details and less of the less important details of the pattern. In terms of our economic example, though they will both imply the same pattern of economic events, other things equal, the conjunction missing the same number of economic data points will imply less of the economic details than another conjunction missing the same number of noneconomic data points. Thus, ceteris paribus, of two otherwise identical conjunctions of parameters and data, both missing the same number of data points, the system missing data for more important parameters will imply predictions of lesser degree than another system missing data for less important parameters.

A bit of reflection, especially upon Hayek's definition of complex phenomena, reveals further dimensions with respect to which the scientist's knowledge may be limited to some extent or other. Moreover, these dimensions concern aspects of the phenomena of prediction that are not easily expressible in terms of more or less, or more or less 
important, knowledge, but instead concern the scientist's knowledge of purely qualitative matters relevant to some complex phenomena.

The theory problem begins with ignorance of at least one of the parameters relevant to the complex phenomena under investigation, but it does not suffice to resolve the problem that the scientist simply enumerate the pertinent variables and understand their relative importance to the phenomena; she must also know something of the kinds of values that each of the parameters can assume. It might seem natural to think that a particular parameter can only take values of the same kind, ontologically speaking-i.e., that one and the same parameter cannot assume, say, either the value red or dog or the number seven. In fact, nothing in Hayek's methodology of sciences of complex phenomena licenses this assumption. However, whether this assumption holds or not, the scientist must know something of the ontological properties of the relevant parameters-either that they each take values of the same kind or that some take values of different kinds. Of course, the scientist may be in a position to know both which of these latter conditions holds and the number of parameters, without knowing whether the values a particular variable assumes are all, e.g., of the kind color, quadruped mammal, positive integer, or some combination thereof. With regard to considerations of predictive degree, the most that can be established with regard to such circumstances is the rather trivial proposition that, other things equal, predictive degree increases as the scientist's theoretical knowledge improves in the relevant sense. That is, the scientist will be able to rule out more events as she improves her knowledge of the relevant properties of the phenomena represented by the parameters of her theory.

But, alas, the theory problem is not merely that of tallying, and identifying both the explanatory importance and ontological properties of, all of the parameters; it extends to that of specifying the interconnections of various subsets of these parameters both with each other and with the external environment. A full resolution of the theory problem requires knowledge of these latter circumstances. Moreover, given Hayek's definition of complex phenomena as those orders that emerge from the internal and external interconnections of a large number of elements, which possess "certain general or abstract features which will recur independently of the particular values of the individual data, so long as the general structure [...] is preserved" 
(Hayek 1967 [1964], 26), and his argument, ${ }^{13}$ that the "cause" of a particular emergent order is the whole network of parameters and particular values from which it emerges, the theory problem includes determining the limits of the constancy of some order given changes in either the values of the data or the interconnections both between various subsets of parameters and between these subsets and the environment. That is, a full solution to the theory problem requires the scientist to consider the extent to which either the values or relevant interconnections of different subsets of parameters can change before the order is supplanted either by some altogether distinct order or by disorder. ${ }^{14}$

Again, philosophical analysis in vacuo is largely impotent to pronounce on the effects of these matters upon considerations of predictive degree beyond the rather trifling claim that, other things equal, the predictive degree of some theoretical system increases-i.e., the occurrence of more events can be ruled out-as the scientist acquires better knowledge of the interconnections both between different subsets of parameters and between these various subsets and the environment; and increases as well as better knowledge is acquired of the manner in which the emergence of some complex phenomena depends on the maintenance of particular interconnections and the values assumed by the parameters.

\section{VI}

It remains to say a few brief words about the sense in which pattern predictions are falsifiable. If one is not careful to read Hayek charitably, it would be too easy to infer an inconsistency between Hayek's methodology and his numerous claims (e.g., at several points in both 1967 [1955] and 1967 [1964]) that pattern predictions are falsifiable. After all, the possibility of a conclusive falsification requires that the scientist of complex phenomena possess knowledge that she cannot possess according to Hayek's methodology, because she is always subject to some extent to either the "data problem" or the "theory problem" (and usually both).

\footnotetext{
${ }^{13}$ See discussion above, pp. 48-49.

${ }^{14}$ Hayek's methodology leaves open the possibility of "feedback" between an order and the phenomena from which it emerges. Thus, a full solution of the theory problem with respect to such an order would also require knowledge of the nature and extent of this feedback.
} 
But, of course, this picture of falsification-which Imre Lakatos (1968-1969, 152-162) once dubbed the "naïve" conception-is untenable in virtually all philosophies of science. According to the naïve view, a single observation is sufficient to conclusively falsify a universal statement. The paradigmatic and oft-quoted example is the eighteenthcentury discovery of black swans in Australia as a purportedly decisive falsification of the universal proposition that "All swans are white". But, as has been pointed out by Duhem (1954 [1906]), Popper (1959 [1934]), and Quine (1961 [1951]), a universal statement can always be saved from an apparent falsification if one is prepared to invoke some ad hoc hypothesis such as, in the case of the apparent observation of Australian black swans, that "The Australian climate causes spectral inversion in bird watchers". As Popper puts it,

[...] no conclusive disproof of a theory can ever be produced; for it is always possible to say that the experimental results are not reliable, or that the discrepancies which are asserted to exist between the experimental results and the theory are only apparent and that they will disappear with the advance of our understanding (Popper 1959 [1934], 50).

Because such ad hoc hypotheses are always available, naïve falsificationism is untenable.

So it is on Hayek's methodology of sciences of complex phenomena. The theory of predictive degree implies that only a precise prediction of a particular event-a prediction of degree 1 -is fully falsifiable in the sense required by naïve falsificationism. Such a prediction rules out all but one of the multitudinous events relevant to the complex phenomena under investigation, i.e., it rules out all ad hoc hypotheses. If the scientist's knowledge were ever so comprehensive as to be able to exclude all but one event, then a falsification would indeed necessitate the rejection of the relevant theory. But, of course, given that Hayek (1967 [1964], 27) defines the sciences of complex phenomena in terms of the presence of (what I am calling) the "data problem", the impossibility of a scientist's knowledge being so total and, thus, the untenability of naïve falsificationism is part and parcel of Hayek's methodology. Naturally, the further possibility of the "theory problem" makes the naïve picture of falsifiability even less plausible. That is, if, as Hayek (1967 [1956], 124) says in "The dilemma of specialization", "all the scientific knowledge we can bring to bear [...] will be only a 
small part of the foundations of our opinions", then this scientific-or, as Hayek (1967 [1964], 29) calls it elsewhere, "theoretical"-knowledge is not necessarily falsified when our opinions are controverted by experience. $^{15}$

So, Hayek was at least not a "naïve" falsificationist. Unfortunately, considerations of brevity prevent me from providing a positive account of the precise nature and extent of Hayek's more "sophisticated" falsificationism in the present context. In particular, I cannot here explicate (my views concerning) the relationship of Hayek's methodology of sciences of complex phenomena to Popper's falsificationism. Suffice it to say here that so long as a conjunction of theoretical parameters and their respective values has a positive predictive degree, it rules out some events-perhaps, in the extreme, only one-and, if some such prohibited event is observed, then this would count as a falsification on Hayek's methodology.

\section{VII}

Hayek used his methodology of sciences of complex phenomena-and, especially, his emphasis upon the limits of knowledge in the social sciences-as a weapon in his well-known debates with advocates of various social schemes that he believed to be epistemologically ill-conceived. Nonetheless, despite their significance for these and other aspects of his scientific and philosophical programs, he never fully explicated the consequences of his methodological arguments.

In an effort to make these implications plain, the present paper has applied what is in essence a method of decreasing abstraction to both Hayek's two-pronged epistemology and his definition of complex phenomena. That is, in section I, we considered the implications for predictive degree of the simplest case where the scientist possesses all of the relevant theoretical knowledge and none of the data. In section II, we dropped the former assumption and considered the degree of pattern predictions where the scientist's theoretical knowledge is also

\footnotetext{
${ }^{15}$ However, it must be said that Duhem-Quine considerations undermine the notion that a stark line can be drawn between sciences that investigate simple phenomena and sciences that investigate complex phenomena. That is, the presence of the data and theory problems that beset the latter sciences demarcates nothing: all sciences are subject to these problems to some extent or other. Of course, that Hayek was aware that no hard-and-fast distinction can ultimately be drawn between complex and simple sciences is reflected in the arguments of his original essays on "Degrees of explanation" (1967 [1955], especially 17-18) and "The theory of complex phenomena" (1967 [1964], especially 24-25).
} 
limited. In section III, we established the impossibility of determining in abstracto the predictive degrees of multiple rival theories, which led to the observation that our theory of predictive degree is itself limited to pattern predictions. In section IV, we dropped the assumption that knowledge of each theoretical parameter (and its datum) is equally relevant to an explanation of some complex phenomena. In section $\mathrm{V}$, we dropped the assumption that the scientist possesses knowledge of the ontological properties of the various parameters as well as knowledge of the interconnections both between the various subsets of parameters and between these subsets and the environment.

This approach highlights different respects, previously unnoticed in the literature on Hayek, in which relevant knowledge might be limited, that extend beyond the fact emphasized by Hayek that the scientist of complex phenomena is often ignorant of some or all of the data relevant to a particular analysis-a difficulty herein christened the "data problem". In particular, the so-called "theory problem"-the possibility that the scientist's ignorance might extend to one or more of the theoretical parameters relevant to a full explanation of some complex phenomena-has been especially emphasized here. A consequence of the theory problem is that predictions can be of lesser degree than that of Hayek's (mere) pattern predictions: partial pattern predictions are possible. Furthermore, the extent of a scientist's ability to fully explain or predict events with precision depends on her knowledge of parameters (and their associated data) that are particularly important to such an explanation. Hayek's conception of complex phenomena also implies that a complete solution of the theory problem-and, thus, the possibility of precise predictions-requires knowledge of a more qualitative variety that is not easily expressible in terms of greater or lesser predictive degrees. But, more than this, the reflexivity of Hayek's emphasis on fragmented and fallible knowledge has been established as a consequence of the possibility of only limited explanations of explanation. Hayek's methodology implies the impossibility of either a complete explanation or a precise prediction of explanations and predictions.

\section{REFERENCES}

Duhem, Pierre. 1954 [1906]. The aim and structure of physical theory. Trans. Philip P. Wiener. Princeton (NJ): Princeton University Press.

Hayek, F. A. 1948 [1935]. The present state of the debate. In Individualism and economic order. Chicago: University of Chicago Press, 148-180. 
Hayek, F. A. 1948 [1940]. Socialist calculation III: the competitive 'solution'. In Individualism and economic order. Chicago: University of Chicago Press, 181-208.

Hayek, F. A. 1948 [1945]. The use of knowledge in society. In Individualism and economic order. Chicago: University of Chicago Press, 77-91.

Hayek, F. A. 1952 [1942-1944]. The counter-revolution of science: studies on the abuse of reason. Glencoe (IL): Free Press. [Originally published in three parts as: Scientism and the study of society. Economica, 9 (35): 267-291; 10 (37): 34-63; 11 (41): 27-39]

Hayek, F. A. 1952. The sensory order: an inquiry into the foundations of theoretical psychology. Chicago: University of Chicago Press.

Hayek, F. A. 1967 [1955]. Degrees of explanation. In Studies in philosophy, politics, and economics. Chicago: University of Chicago Press, 3-21.

Hayek, F. A. 1967 [1956]. The dilemma of specialization. In Studies in philosophy, politics, and economics. Chicago: University of Chicago Press, 122-132.

Hayek, F. A. 1967 [1964]. The theory of complex phenomena. In Studies in philosophy, politics, and economics. Chicago: University of Chicago Press, 22-42.

Hayek, F. A. 1967. Notes on the evolution of systems of rules of conduct. In Studies in philosophy, politics, and economics. Chicago: University of Chicago Press, 66-81.

Hayek, F. A. 1978 [1975]. The pretence of knowledge. In New studies in philosophy, politics, economics, and the history of ideas. Chicago: University of Chicago Press, 23-34.

Hayek, F. A. 1991 [1920]. Contributions to a theory of how consciousness develops. Trans. Grete Heinz. Hoover Institution, Hayek Archives, box 92, folder 1.

Hayek, F. A. 1995 [1931a]. Reflections on the pure theory of money of Mr. J. M. Keynes. In The collected works of F. A. Hayek, ed. Stephen Kresge, vol. 9, Contra Keynes and Cambridge: essays, correspondence, ed. Bruce Caldwell. Chicago: University of Chicago Press, 121-146.

Hayek, F. A. 1995 [1931b]. A rejoinder to Mr. Keynes. In The collected works of F. A. Hayek, ed. Stephen Kresge, vol. 9, Contra Keynes and Cambridge: essays, correspondence, ed. Bruce Caldwell. Chicago: University of Chicago Press, 159-164.

Hayek, F. A. 1995 [1932]. Reflections on the pure theory of money of Mr. J. M. Keynes (continued). In The collected works of F. A. Hayek, ed. Stephen Kresge, vol. 9, Contra Keynes and Cambridge: essays, correspondence, ed. Bruce Caldwell. Chicago: University of Chicago Press, 174-197.

Hayek, F. A. 2014 [1961]. A new look at economic theory. In The collected works of F. A. Hayek, ed., Bruce Caldwell, vol. 15, The market and other orders. Chicago: University of Chicago Press, 373-426.

Keynes, John Maynard. 1971 [1930]. A treatise on money: the pure theory of money. In The collected writings of John Maynard Keynes, ed. Donald Moggridge, vol. 5. London: Macmillan.

Keynes, John Maynard. 1973 [1931]. The pure theory of money: a reply to Hayek. In The collected writings of John Maynard Keynes, ed. Donald Moggridge, vol. 13, The general theory and after, part I: preparation. London: Macmillan, 243-256.

Lakatos, Imre. 1968-1969. Criticism and the methodology of scientific research programmes. Proceedings of the Aristotelian Society, 69: 149-186.

Pareto, Vilfredo. 1927. Manuel d'économie politique [2nd ed.]. Paris: M. Giard.

Polanyi, Michael. 1966. The tacit dimension. Garden City (NY): Doubleday \& Company, Inc. 
Popper, Karl. 1959 [1934]. The logic of scientific discovery. London: Hutchinson.

Ryle, Gilbert. 1946. Knowing how and knowing that. Proceedings of the Aristotelian Society, 46: 1-16.

Quine, W. V. O. 1961 [1951]. Two dogmas of empiricism. In From a logical point of view [2nd revised ed.]. New York: Harper and Row. [Originally published in The Philosophical Review, 60: 20-43]

Scott Scheall teaches in the science, technology, and society department at Arizona State University. He received his $\mathrm{PhD}$ in philosophy from Arizona State in 2012. He is a former research fellow with Duke University's Center for the History of Political Economy and, during the current academic year (2014-2015), is a postdoctoral fellow with the F. A. Hayek program for advanced study in philosophy, politics, and economics at George Mason University. Scott is co-editor of Research in the History of Economy Thought and Methodology.

Contact e-mail: <scott.scheall@asu.edu> 